\title{
Les modalités énonciatives de la reprise du discours d'autrui dans les écrits de recherche et les écrits didactiques
}

\author{
Bertrand Daunay* \\ Isabelle Delcambre**
}

\begin{abstract}
Résumé
L'article vise à décrire les modalités énonciatives des reprises du discours d'autrui effectuées dans des écrits académiques. Sont comparés deux types d'écrit produits par des enseignants-chercheurs : des écrits didactiques, élaborés pour des cours à distance, et des écrits de recherche, à destination de la communauté scientifique. Par modalités énonciatives, il faut entendre les modes de prise en charge, par un énonciateur, d'un discours d'autrui qu'il reprend dans son propre discours. Après avoir présenté leur cadre théorique et la terminologie qu'ils proposent (notamment l'identification de deux pôles, nommés « paraphrase » et " métaphrase », opposant les modalités énonciatives de la reformulation), les auteurs décrivent leur corpus et leur méthodologie avant de présenter leurs résultats - dont le plus net : la part respective de ces deux modalités diffère selon le contexte de production (écriture d'enseignement ou écriture de recherche).
\end{abstract}

Mots-clés: Énonciation. Discours d'autrui. Paraphrase. Métaphrase. Didactique. Écrits académiques.

1 Univ. Lille, EA 4354 - CIREL - Centre interuniversitaire de recherche en éducation de Lille, F-59000 Lille, France.

2 Univ. Lille, EA 4354 - CIREL - Centre interuniversitaire de recherche en éducation de Lille, F-59000 Lille, France. 
L'énoncé d'auteur, au moment d'intégrer dans sa composition un autre énoncé, élabore des normes syntaxiques, stylistiques et compositionnelles pour l'assimiler partiellement, pour l'associer à l'unité de l'énoncé d'auteur, tout en conservant, au moins sous une forme rudimentaire, l'autonomie originelle (syntaxiques, compositionnelles, stylistiques) de l'énoncé d'autrui, faute de quoi ce dernier ne pourrait être appréhendé dans sa plénitude. (VOLOŠINOV, 1929/2010, p. 365)

Dans les travaux sur les littéracies universitaires ou académiques ${ }^{1}$, la question de la reprise du discours d'autrui dans un texte, forme spécifique d'interaction entre l'écriture et la lecture ${ }^{2}$, a une place centrale ${ }^{3}$. C'est dans cette lignée que s'inscrit notre travail ${ }^{4}$, qui veut aborder cette question d'une manière spécifique, en traitant les modalités énonciatives de la reprise du discours d'autrui dans des écrits d'enseignants-chercheurs. Plus précisément, il s'agit pour nous de comparer les modalités qu'ils mettent en œuvre dans deux types de discours académique qu'ils ont à produire: le discours de recherche (entendu, comme « discours produit dans le cadre de l'activité de recherche à des fins de construction et de diffusion du savoir », pour reprendre la définition de l'écrit scientifique par BOCH, RINK, 2010, p. 5) et le discours didactique (que nous entendrons ici comme discours produit dans le cadre de l'activité d'enseignement à des fins de présentation d'un savoir par un professeur à des étudiants) ${ }^{5}$.

Nous parlons ici de modalités énonciatives pour désigner les modes de prise en charge, par un scripteur, d'un discours d'autrui qu'il reprend dans son propre discours. Si, pour reprendre la définition de LE QUERLER (1996, p. 61), la modalité est « l'expression de l'attitude du locuteur par rapport au contenu

1 Le mot « universitaire » traduit en français le terme anglais academic. Il est généralement préféré au terme « académique » qui, en français, porte des connotations souvent négatives (DELCAMBRE, DONAHUE, 2012, p. 135-136). "Littéracies universitaires » est donc un équivalent de Academic Literacies.

2 Problématique didactique ancienne (cf. REUTER, 1994) qui reste pertinente, notamment pour penser les questions de formation (cf. DAUNAY, DELCAMBRE, DUFAYS, THYRION, 2007) et, plus récemment, l'acculturation aux littéracies universitaires (cf. NELSON, CASTELLÓ, 2012

3 Ce texte est écrit en respectant les normes de l'orthographe rectifiée.

4 Réalisé dans le cadre du projet CAPES-COFECUB, « Le discours universitaire dans la recherche et l'enseignement : questions autour de l'appropriation de la parole d'autrui » (2014-2018), dirigé par Juliana Assis et Sophie Bailly. Un premier état de notre travail a été présenté dans DAUNAY, DELCAMBRE (2016), ainsi que dans une conférence que nous avons présentée à Belo Horizonte (université de PUC Minas) le 3 juin 2016. Merci aux collègues et aux étudiants pour leurs retours, qui nous ont permis d'affiner notre présentation. Merci également à Françoise Boch et Francis Grossmann pour leur lecture attentive de nos premiers écrits qui nous a permis de préciser notre approche théorique. 5 Nous renvoyons au travail de caractérisation du discours didactique et du discours de recherche (mais aussi du discours de vulgarisation réalisé naguère par BEACCO, DAROT (1984). 
propositionnel de son énoncé », les modalités énonciatives dont nous parlons ici sont doublement spécifiques : elles concernent l'énoncé d'autrui inséré dans le discour0s, et elles se limitent aux modes de prise en charge de cet énoncé par le scripteur.

Plus précisément encore, nous visons à identifier le degré de cette prise en charge énonciative du discours d'autrui par un auteur dans son texte ; pour cela, nous avons identifié deux pôles, représentés respectivement par ce que nous appelons «métaphrase » et " paraphrase » : dans un cas, le discours du scripteur ${ }^{6}$ prend en charge totalement le propos d'autrui, en en devenant l'énonciateur, pour ainsi dire ; à l'autre pôle, c'est le discours d'autrui qui, en quelque sorte, gouverne l'énonciation dans le discours qui le reprend et dont l'énonciateur s'efface alors. Ces modalités de prise en charge énonciative du discours d'autrui sont une des formes rhétoriques de la subjectivité du scripteur qui montre par-là, de manière plus ou moins visible, son positionnement par rapport au discours d'autrui (cf. la mise au point de FLOREZ, 2013, à propos de la reformulation et de la citation positionnée).

Nous faisons ci-après une mise au point théorique qui précisera la manière dont nous posons le problème, qui s'inscrit dans une tradition déjà ancienne, depuis la description des formes de modalisation de "l'hétérogénéité montrée » par AUTHIER-REVUZ (1982) jusqu'à l'identification du « discours représenté » (représentant aussi bien la forme que le contenu du dire étranger) que met en avant la ScaPoLine (NØLKE, FLØTTUM, NORÉN, 2004). L'originalité de notre approche tient à ce qu'elle est davantage centrée sur une distinction, qui s'avèrera intéressante, on le verra, entre les «formes » et les «modalités » de la reprise du discours d'autrui.

Notre hypothèse est que, s'agissant des modalités de reprise du discours d'autrui, le scripteur ne met pas en œuvre tout à fait les mêmes normes selon les contextes de production écrite. Nous posons que dans les écrits didactiques, la paraphrase devrait l'emporter sur la métaphrase, dans la mesure où, dans ces écrits, est mise en avant la présentation des auteurs à faire découvrir aux étudiants ; dans les textes scientifiques au contraire, la métaphrase l'emporterait sur la paraphrase, dans la mesure où c'est l'argumentation du scripteur qui l'emporte. 
Si les outils de notre description sont essentiellement linguistiques, notre approche est didactique, pour deux raisons : d'une part, nous envisageons l'écrit didactique comme un écrit professionnel des enseignants, qui met en scène des sujets didactiques (cf. DAUNAY, 2011) et cette définition seule engage une certaine description de l'objet. D'autre part, comme le discours didactique de l'enseignant exhibe des manières de faire en matière de discours académique, ses proximités ou ses distances avec le discours de recherche n'est pas sans intérêt dans la perspective d'une réflexion sur l'acculturation des étudiants aux modalités énonciatives de la reprise du discours d'autrui dans les textes qu'ils lisent comme dans ceux qu'ils écrivent (cf. BOUCHARD, PARPETTE, 2007). Enfin, les catégories d'analyse que nous proposons peuvent servir de cadre pour analyser les difficultés des étudiants et guider l'aide qu'on peut leur apporter ${ }^{7}$.

\section{Métaphrase et paraphrase : précisions théoriques}

\section{Première approche}

$\mathrm{Si}$ « paraphrase » est bien connu, « métaphrase » est peu attesté en linguistique (et on ne le trouve pas dans la plupart des dictionnaires de sciences du langage). Notre choix de ces deux termes, qui font système de par leur similitude dérivationnelle, tient au fait qu'on peut voir dans le préfixe para- l'expression de la proximité énonciative entre texte cité et texte citant, et dans le préfixe méta-, l'expression de la distance (le texte citant ne suivant pas l'énonciation du texte cité $)^{8}$. Aussi nous emparons-nous de « métaphrase » pour référer aux cas où l'énonciation du texte-source est quasi effacée, absorbée dans celle du texte qui le reprend, à l'opposé de la paraphrase qui mime l'énonciation du texte-source'. Mais il ne faut pas voir dans le préfixe méta- le

7 Dans la lignée de travaux anciens en didactique, particulièrement représentés dans les équipes de recherche de Grenoble et de Lille ; pour simples illustrations, cf. BOCH, GROSSMANN (2002) ; DABÈNE (1987) ; DELCAMBRE (2001); FRIER (1998); REUTER (2004).

8 Le sens donné à ces préfixes suit l'usage du grec ancien. Précisons cependant que paraphrasis et metaphrasis ont des sens assez proches dans l'Antiquité et signifient en gros « reformulation ». Ces deux mots diffèrent par le fait que la paraphrase serait stylistiquement plus élaborée que la métaphrase, laquelle consisterait en un simple changement de mots (cf. Roberts, 1985, p. 26). Notons que, à part Mounin (1974/1993, s.v.), qui cite " métaphrase » en renvoyant à " paraphrase », aucun des dictionnaires suivants n'a d'entrée « métaphrase » : BUSSMANN (1996) ; CRYSTAL (1980/1997); DUBOIS et al. (1994/2012); NEVEU (2004/2011).

9 Cette opposition a été à l'origine travaillée par B. Daunay dans ses recherches sur le commentaire de texte (DAUNAY, 2002): il opposait alors paraphrase à détextualité. Nous avons présenté ailleurs 
signe que la métaphrase serait plus métalinguistique que la paraphrase ${ }^{10}$.

Prenons deux exemples contrastés pour illustrer nos définitions. Dans un cas, imaginons un exergue ou un diaporama où le scripteur reproduit une citation d'auteur sans l'introduire, sans qu'elle soit même encadrée par un énoncé du scripteur ; en ce cas, c'est comme si l'auteur prenait la place du scripteur, qui disparait, pour ainsi dire, à cet endroit du texte. Nous parlons alors de «paraphrase ». Imaginons à l'inverse que l'auteur d'un mot ou d'un syntagme, jugé particulièrement familier aux lecteurs pour ne pas demander de référence exacte, mais marqué quand même comme « hétérogène », issu d'une autre « voix » (en général, ce sont des italiques ou des guillemets que l'on emploie pour le signaler), soit incorporé dans le texte du scripteur, avec parfois une modification de forme ou de sens : en ce cas, si la voix de l'auteur est audible, l'énonciation qui domine est celle du scripteur qui prend en charge la reprise. C'est ce que nous appelons « métaphrase $»^{11}$.

Précisons que les phénomènes que nous traitons sont locaux (au niveau phrastique ou interphrastique) et ne concernent que l'" hétérogénéité montrée " (AUTHIER-REVUZ, 1982), car le but est bien d'identifier la manière dont, sur le plan énonciatif, le discours d'autrui est montré comme intégré au discours du scripteur. L'analyse ne convient donc pas aux cas de plagiat ou d'ignorance par le scripteur de l'origine de tel ou tel mot ou concept, de même qu'elle ne concerne pas la polyphonie structurelle de tout discours, chaque mot pouvant être considéré, d'un certain point de vue, comme celui d'autrui (cf. BAKHTINE, 1934/1978)...

(DAUNAY, DELCAMBRE, 2016 ; DAUNAY, 2017) les raisons du choix de substituer « métaphrase » à « détextualité » et les différences entre les deux phénomènes (renouvelons ici nos remerciements à Daniel Bart pour les discussions qui nous ont permis de clarifier le système conceptuel que nous proposons ici).

10 C'est dans le sens de «phrase métalinguistique» que " métaphrase» a été utilisé dans quelques rares écrits linguistiques, à la suite de DOMINICY (1984, p. 192), qui l'utilise au sens de la reformulation d'un énoncé que propose le grammairien pour rendre compte de l'adéquation de son analyse théorique à l'énoncé.

11 Le discours d'autrui devient «un support de l'argumentation» du scripteur, pour reprendre les termes de Doury (2004) qui ajoute : «l'argumentation au niveau de l'échange enchâssant est inextricablement mêlée à l'argumentation "racontée", telle qu'elle se déroule au sein de l'échange enchâssé, au point que les deux peuvent se fondre en une argumentation aux coordonnées énonciatives floues» (ibid., p. 263). Cette analyse du discours rapporté dans les échanges oraux est très proche de ce que nous appelons «métaphrase». 


\section{Quelques exemples nets de métaphrase}

Présentons quelques cas nets qui permettent de différencier les deux pôles, en puisant nos exemples dans notre corpus, que nous présentons plus loin. Pour la lisibilité de ce qui suit, précisons ici le codage : chaque extrait est désigné par une majuscule référant à l'enseignant $(\mathrm{A}, \mathrm{B}, \mathrm{C}, \mathrm{D})$, suivie d'une minuscule référant au corpus ( $r$ pour recherche, d pour didactique) et d'un chiffre identifiant le document (pour le corpus d, le niveau est également précisé : L pour licence, M pour master).

Commençons par un exemple emblématique de métaphrase:

Une perspective de recherche didactique pourra se donner les moyens conceptuels et méthodologiques de construire l'intérêt heuristique des erreurs des élèves (Astolfi, 1997), leurs silences (Zaid, 2012), leurs imbécilités (Daunay, 2014) ou les «dysfonctionnements » de leurs réponses (Reuter, 2013b) en ce qu'ils constituent des indices concernant les stratégies, les connaissances disciplinaires, les difficultés et obstacles que rencontrent les élèves ou d'ailleurs les problèmes que posent les tests et questions auxquels ils sont soumis. [Br1]

L'auteur construit une argumentation présentée comme personnelle, en incluant dans son propos de nombreuses expressions d'autres auteurs qui ne les avaient pas forcément conçues dans cette logique ni envisagées dans ces rapprochements, ni même utilisées sous cette forme (c'est le cas de la flexion du mot original: le scripteur parle d'«imbécilité», alors que l'auteur cité n'emploie pas ce mot mais celui d'«imbécile», qu'il construit comme figure théorique).

Ce type de "discours multiréférencé met au premier plan une voix d'auteur », comme le dit GROSSMANN (2011, p. 208), qu'il juge caractéristique des discours scientifiques, et qui montre bien la dimension métaphrastique du discours, pris en charge par le scripteur, " les autres voix étant réduites au rang d'emblèmes de position ou, parfois, attachées à un développement sous la forme d'énoncés reformulant un point de vue » (ibid.). GROSSMANN signale ailleurs (2002) le problème spécifique que pose un tel « discours multiréférencé », en des termes qui ne sont pas sans évoquer notre traitement de la métaphrase:

L'hétérogénéité, certes, est montrée, et même revendiquée, mais l'avalanche des noms propres, à rebours de la problématique habituelle des théories de la polyphonie, pose la question de 
l'unicité : comment s'effectue l'intégration dans un discours «unique», cohérent, de ce qui est par définition indexé sur le multiple? (Grossmann, 2002, p. 225).

Un autre exemple de métaphrase se trouve dans le passage suivant où une seule référence est en jeu, mais insérée dans une argumentation qui est celle du scripteur, ce qui lui permet de tisser à sa manière le discours d'autrui:

Mais, comme dirait Sensevy (2007), comprendre l'action de l'enseignant c'est d'abord comprendre comment le contenu propre à cette action la spécifie, comment le contenu en spécifie en particulier l'évolution. [Ar3]

L'expression « comme dirait Sensevy », qui ouvre la reformulation, montre (par l'adverbe « comme » et le conditionnel), que le scripteur s'approprie le propos qu'il insère dans le sien propre ${ }^{12}$.

Évoquons encore, comme exemples typiques de métaphrase, ces passages où le texte dit « ce que ne dit pas » le discours d'autrui, qui nous ont semblé des exemples assez nets de métaphrases, comme celui-ci:

Lahire ne parle pas spécifiquement des schèmes d'utilisation, mais ceux-ci sont bien, pour Vergnaud comme pour Rabardel, « relatifs à une classe de situations » (Vergnaud, 1991). [Cr3]

Ou encore ceux où le scripteur reprend les mots d'un auteur, mais les met en cause de manière polémique, comme:

Ce travail nous a ainsi amené dans un premier temps à montrer comment cette approche de la littératie dans le PISA et son opérationnalisation dans des épreuves dites " authentiques » tendent à instituer, un soi-disant « Réel » universel comme contenu d'évaluation et à rendre conjointement moins visibles les savoirs disciplinaires en jeu. [Brl]

Ces exemples sont pour nous des métaphrases assez claires, dans la mesure où le scripteur prend en charge à ce point le discours de l'autre qu'il prend de nettes distances avec lui tout en l'insérant dans son propre propos.

12 Notons à cet égard que l'expression «pour paraphraser » introduit un énoncé qui est l'exact contraire de ce que nous nommons ici paraphrase... Par exemple : « Les EPA, pour paraphraser (Rabardel 1995), p. 12 ne sont pas donnés à l'utilisateur, celui-ci les élabore progressivement » [Cr3]. En effet, le scripteur reprend les mots d'un auteur, mais en les associant à un tout autre contexte et pour un autre sens, géré par le scripteur seul. 


\section{Quelques exemples nets de paraphrase}

Avec la paraphrase, l'énonciation est à l'inverse laissée à l'auteur représenté, comme typiquement dans les citations entre guillemets : la reproduction à l'identique d'un extrait du discours d'autrui « fait retentir la lecture dans l'écriture » (pour reprendre le mot de Compagnon (1979, p. 27), fait retentir aussi l'énonciation du texte-source. Inutile d'en donner des exemples, tant le procédé est connu. Prenons comme un autre exemple typique de la paraphrase ce que d'aucuns appellent le « discours direct libre » (voir par exemple ROSIER, 1999). :

Le problème est :

- subjectif : un point de vue, une intention ;

- temporel : une solution après (une promesse) ;

- spatial : un cotexte (le problème n'est pas seulement dans ma tête, il est dans le monde aussi). [AdM1]

Ce qui est souligné en gras est pour nous une paraphrase, où la voix d'autrui est insérée dans le texte en conservant son propre système d'énonciation, au point de faire coexister deux sources du discours différentes, deux « je » différents ; autrement dit, le discours du scripteur s'efface pour laisser la place au discours d'autrui ${ }^{13}$. Dans ce cas, il s'agit d'une voix reconstruite, inventée, qui semble typique d'un certain point de vue, attesté dans les données étudiées par le scripteur. Plus rare est le cas où ce type de reprise concerne un auteur, dans la mesure où c'est une modalité énonciative ou stylistique qui n'est pas de mise dans le discours académique. En voici cependant un exemple:

Pour certains, la réponse est simple : à rien ! C'est notamment la position de Célestin Freinet. [DdM3]

Mais outre ces deux formes de discours directs, toutes les reprises (au discours direct, indirect, indirect libre, narrativisé, etc.) sans interventions du scripteur peuvent relever de la paraphrase.

Pour résumer, avec la métaphrase, on peut dire que le discours du scripteur colore, en le modifiant au plan énonciatif, le discours de l'auteur. C'est l'inverse de

13 DAUNAY (2002) proposait d'appeler paraphrase stricto sensu une reprise qui use du même système énonciatif que le texte-source, au contraire de la paraphrase largo sensu, qui a certaines des caractéristiques de la première, mais modifie l'énonciation du texte-source. 
la paraphrase, où l'énonciation du discours d'autrui colore, sur le plan énonciatif, le discours qui le reprend.

\section{Établissement d'un corpus et d'un document de recherche}

Nous présentons ici en détail notre méthodologie, de la constitution de notre corpus à celle d'un « document de recherche » (cf. DELCAMBRE, LAHANIERREUTER, 2003), élaboré à partir de ce corpus, qui est le résultat de deux opérations : la détermination des reprises dans le corpus ; l'identification des diverses catégorisations de reprises.

\section{Constitution du corpus}

L'étude que nous proposons se fonde sur un corpus construit de manière empirique, à partir de ce qu'ont accepté de nous donner quatre collègues proches. Ils sont tous quatre didacticiens : notre seule variable étant la différence entre écrits de recherche et écrits didactiques, nous avons préféré choisir une seule discipline pour établir cette comparaison. Cela présente un avantage non négligeable, car la connaissance des textes-sources des reprises est souvent nécessaire dans le cadre de notre enquête, même si nous ne nous plaçons pas dans la perspective génétique de DOLIGNIER (2016) : quand nous avons recours aux textes-sources, c'est pour confirmer ou infirmer l'identification des modalités, non pour évaluer le « degré de dépendance » des écrits analysés au texte d'autrui (ibid. p. 134).

Pour des raisons de faisabilité mais aussi pour faciliter la comparaison, nous avons choisi, comme écrits didactiques, des textes rédigés ou des diaporamas constituant des cours magistraux réalisés dans le cadre de l'enseignement à distance en ligne. Les productions de recherche retenues sont des articles dans des revues, des chapitres d'ouvrages ou des communications rédigées. Tous les textes des deux corpus ont été rédigés en 2014-2015.

Il importe sans doute de savoir que A et D enseignent en ESPE (Écoles supérieures du professorat et de l'éducation), tandis que $\mathrm{B}$ et $\mathrm{C}$ enseignent à l'université, dans un département de sciences de l'éducation. Malheureusement, le hasard veut que les cours de licence (L3) de notre corpus aient été donnés à 
l'université et ceux de master (M1 et M2) à l'ESPE ; nous ne pourrons donc pas savoir ce qui fait la spécificité du niveau ou du lieu d'enseignement. Il faudrait pour cela étendre le corpus.

Après une sélection faite sur des critères pratiques (par exemple, nous n'avons pas retenu les notes de lecture, ni les articles collectifs, ni les textes en langues étrangères) et de faisabilité (ainsi, nous n'avons retenu que trois écrits de recherche et trois écrits didactiques pour chaque auteur du corpus), nous aboutissons à un corpus de plusieurs centaines de pages ou, plus objectivement, de 1257184 signes (191 491 mots), réparties de façon équilibrée entre le corpus des écrits didactiques et le corpus des écrits de recherche, de même qu'entre les scripteurs.

\section{Détermination des reprises dans le corpus}

Après l'établissement du corpus, nous avons commencé l'opération d'identification des reprises d'autrui, en fonctionnant de manière intuitive, tout en nous donnant l'assurance que notre intuition était raisonnable... En effet, nous avons analysé, tous les deux, séparément, les mêmes documents, en identifiant ce qui était pour nous la reprise d'un discours d'autrui. Nous avons confronté nos relevés et ajusté nos approches intuitives, sans pour autant nous donner des indicateurs définitifs : ce qui n'était pas nécessaire vu la quasi-évidence de la reconnaissance des passages concernés (même si certaines difficultés ont été rencontrées, dont il ne nous est pas possible de rendre compte ici). Nous nous sommes ensuite partagé la tâche et avons analysé chacun la moitié du corpus.

Nous avons isolé en règle générale des segments correspondant à des propositions entre deux ponctuations fortes, autrement dit les signes de ponctuation suivis d'une majuscule ou encore le point-virgule. Nous avons, selon le contexte, considéré le double point comme une ponctuation forte (cf. DRILLON, 1991). Nous n'avons pas appliqué notre règle de segmentation aux énumérations ni aux citations constituées de plusieurs propositions. Précisons enfin que nous avons dû, pour rester cohérents avec nos choix, rétablir parfois (rarement à vrai dire) une ponctuation normée dans le corpus.

Notons que nous considérons aussi comme discours d'autrui les références à « soi comme un autrui », quand un auteur cite un travail dont il est lui-même l'auteur. Par exemple: 
Dans une perspective didactique critique proche, nous avons de notre côté procédé à des analyses exploratoires d'items (Scripteur, Coscripteur, à par.) en vue de mieux décrire l'assise conceptuelle du PISA autour de la notion de littératie. [Br1]

Nous avons choisi de considérer comme paroles d'autrui les paroles anonymes et ou collectives, quand leurs sources étaient clairement identifiées, qu'il s'agisse de courants théoriques ou de propos d'informateurs d'une enquête empirique, comme respectivement dans ces exemples:

Des chercheurs développent à partir des années 1980, une théorisation et une opérationnalisation de ce processus de prise d'information vers l'évaluation formative. [BdL1]

Il est clair en effet pour nos interlocuteurs que les supports budgétaires et les opportunités contractuelles pour les doctorants SHS sont inférieurs à ceux dont disposent les autres disciplines. [Br4]

Il en est de même des formules comme " on appelle ", " on nomme », " on dit », etc., bien mises en valeur dans le discours magistral oral à l'université par CLAUDEL (2011, p. 197-200), comme dans cet exemple:

Le point de vue omniscient (ce qu'on appelle aussi à la suite du critique Gérard Genette la focalisation zéro). [DdM1]

En revanche, nous ne retenons pas une phrase comme " Ces variables sont appelées qualitatives » [CdL5] : le passif, ici, ne fait que permettre au scripteur de donner une information sur une dénomination sans référence précise à une communauté identifiée ou à un auteur. On ne retient pas davantage les passages où les propos rapportés sont clairement ceux que pourrait tenir le scripteur ; par exemple:

Ce n'est pas du tout la même chose de dire que la population est "les élèves de la classe" ou "les notes obtenues par les élèves de la classe". [CdL5].

Précisons encore que nous avons retenu ce que nous appelons les « faux discours d'autrui », c'est-à-dire les discours inventés, parfois nécessaires pour assoir un discours de démonstration (peu nombreux, ils se trouvent plutôt dans 
les discours didactiques) $)^{14}$; nous n'avons retenu que ceux qui sont référés à des énonciateurs fictifs (clairement désignés ou non mais identifiables ${ }^{15}$ ), qui reprennent clairement des paroles prononçables par un énonciateur ayant une certaine autonomie par rapport au scripteur. Voici un exemple (ici, en gras) de ces « faux discours d'autrui » que nous retenons:

Par exemple les élèves peuvent aimer ou non un contenu ou une matière scolaire (j'aime pas les fractions, j'aime bien la poésie....). [CdL1]

Dans la mesure où nous cherchions à identifier les discours d'autrui explicitement posés comme tels par le scripteur, il n'y avait pas trop de difficulté à obtenir un relevé objectif, ce que notre protocole de travail nous a permis, nous semble-t-il, de contrôler au mieux.

\section{Identification des natures et des formes de ces reprises}

Une fois établi ce relevé des reprises, nous avons construit un tableau qui reprenait, en les numérotant, les énoncés repérés (au nombre de 1337). Alors pouvait commencer la catégorisation des reprises, que nous avons, là encore, faite séparément sur deux extraits de corpus avant confrontation et généralisation sur l'ensemble du corpus. Nous avons classé les reprises selon trois critères : leurs natures ; leurs formes ; leurs modalités.

Donnons quelques précisions sur ces catégorisations. Ce que nous appelons nature des reprises correspond à leur source (auteurs académiques ou données de recherche). Ce choix de distinguer ces deux sources nous a semblé intéressant et a effectivement produit quelques résultats, même s'il n'était pas évident et a posé quelques problèmes de délimitation - problèmes qui peuvent expliquer que, dans son approche, GROSSMANN (2002, p. 225) exclue cette source dans son analyse du discours d'auteur.

14 À propos de ce type de propos fictif dans le discours d'enseignants, cf. CLAUDEL (2001, p. 201 203).

15 Dans le cas contraire, on aurait dû prendre en compte, ce que nous n'avons pas fait, tout ce qui relève de la connotation autonymique (REY-DEBOVE, (1978/1997, p. 253), comme : «Parfois, en effet, on peut mener des analyses "à côté de la plaque" " [CdL5]. 
Pour identifier la forme des reprises, nous nous sommes référés à la typologie de BOCH et GROSSMANN (2002), en la modifiant légèrement. Ces derniers distinguent quatre formes : la citation (marquée par les guillemets ou l'italique) ; la reformulation (reprise d'un « dit d'autrui », selon les termes de GROSSMANN, 2002); l'évocation ; l'îlot citationnel.

La distinction entre reformulation et évocation peut être parfois difficile. Nous considérons comme évocation les discours attribués globalement à un auteur ou à un courant théorique dont le scripteur ne précise pas le contenu (c'est au lecteur d'aller en prendre connaissance par lui-même). C'est particulièrement net quand il $\mathrm{y}$ a des références multiples, comme ici:

En outre, dans une perspective qui évacue toute appropriation immédiate, le rapport de l'élève à ce contenu est un rapport d'objectivation (Freitag, 1986, Lenoir, Ibid.). [Ar3]

Nous considérons aussi comme évocation les passages où l'identification condensée du thème du discours d'autrui n'est pas accompagnée de précisions sur ce contenu:

Pour ceux que cela intéresse, l'article «Aux origines de la « science des examens » (1920-1940)» de Jérôme Martin explique l'histoire de cette discipline de recherche. [BdL4]

Nous posons que cette thématisation (en gras), toute rhétorique, diffère d'une reformulation. Mais il est clair que la limite est assez difficile à trancher nettement et c'est souvent l'intuition (contrôlée, comme nous l'avons signalé plus haut) qui permet de dire quand le degré de développement de la thématisation fait basculer de l'évocation à la reformulation. Autre cas limite : quand l'empan de la reformulation n'est pas clair (malgré les vérifications de la source) et que l'on ne sait pas si l'auteur est évoqué ou reformulé, autrement dit, quand cette décision dépend encore du lecteur, on parlera d'évocation. Par exemple:

Il s'agit souvent pour l'enseignant de présenter une image de sa classe qui satisfasse au mieux ces personnes extérieures (Leclercq et al., 2004). [BdL2] 
La quatrième forme, l'îlot citationnel, nous a posé un problème spécifique. Selon GROSSMANN (2002), qui a proposé cette catégorie ${ }^{16}$, l'îlot citationnel consiste à absorber le discours cité dans le discours citant, " en effaçant leurs frontières énonciatives (mais en conservant les marques typographiques distinctives) », ce qui «met en valeur l'expressivité d'un terme ou d'une expression, ainsi détachés du cotexte dans lequel ils sont insérés » (ibid. : 259). Ce qui nous a amenés, dans notre propre enquête, à modifier cette catégorie, c'est que GROSSMANN signale que l'îlot citationnel peut être « inclus ou non dans une reformulation »; or il nous a semblé que ce n'était pas la même chose d'inclure dans le discours citant un segment cité selon qu'il était ou non inclus dans une reformulation. Aussi avonsnous, à la place de l'îlot citationnel, identifié deux catégories de formes :

- l'emprunt : un mot ou un syntagme, référé clairement à un nom d'auteur, inclus dans un segment qui ne se présente pas comme une reformulation ${ }^{17}$;

- l'hybridation : une reformulation qui inclut des syntagmes cités (entre guillemets ou en italique) de l'auteur d'origine.

\section{Identification des modalités}

Ces catégorisations des natures et des formes de reprises ont posé peu de problèmes, car il s'agit de catégories « discrètes »; l'identification des modalités était plus délicate car il s'agit là d'une catégorie "scalaire »: les deux pôles extrêmes que sont la métaphrase et la paraphrase sont reliés par un continuum, qui rend compte de la grande variation des degrés de prise en charge énonciative par le scripteur ou par autrui. Ainsi, il nous a paru nécessaire d'établir une échelle avec seulement cinq degrés pour caractériser les énoncés et identifier des tendances à l'œuvre selon les textes :

16 Qu'il a construite à partir de la notion d'îlot textuel, issue des travaux de Jacqueline AuthierRevuz, dans un sens proche de celui qu'elle donne à ce terme, c'est-à-dire celui d'une « modalisation autonymique » dans laquelle un mot, un syntagme ou un élément textuel plus large, mis entre guillemets (ou à l'oral décroché par l'intonation) est utilisé à la fois en usage et en mention et est en quelque sorte « absorbé » dans le discours citant, ce qui crée souvent un effet particulier, avec ambigüité possible sur le statut (modalisation distanciante ou effet d'une présence « autre », comme dans le discours journalistique qui intègre des éléments cités dans un discours propre). (Communication personnelle de Francis Grossmann). Ajoutons que l'on trouvera une mise au point récente sur l'îlot textuel dans FLECK (2015).

$17 \mathrm{La}$ référence à un auteur est nécessaire pour figurer dans notre relevé. Cela pourrait se discuter quand un mot, nettement issu d'un auteur, est repris ensuite dans le texte avec ou sans marques de mention (guillemets ou italique). Mais nous nous en sommes tenus à la règle de la référence coprésente pour éviter les zones de flou. 


\section{Figure 1 - Échelle entre métaphrase et paraphrase}

$\mathrm{P}++\quad=$ ce qui est clairement de la paraphrase (le discours cité gouverne l'énonciation)

$\mathrm{P}+\quad=$ ce qui penche vers la paraphrase

$\mathrm{P}$ ou $\mathrm{M}=$ ce qui est indécidable

$\mathrm{M}+\quad=$ ce qui penche vers la métaphrase

$\mathrm{M}++\quad=$ ce qui est clairement de la métaphrase (le discours citant gouverne l'énonciation)

Nous n'avons pas construit une liste exhaustive des marqueurs des modalités des énoncés mais, dans le cours de notre analyse, nous en avons retenu certains qui pouvaient garantir une certaine régularité dans leur identification. Nous avons indiqué plus haut, dans notre première approche de la métaphrase et de la paraphrase, quelques-uns de ces marqueurs qui permettaient de différencier les deux pôles extrêmes de l'échelle.

Ainsi, la référence, dans la même phrase du scripteur, à plusieurs auteurs ou à plusieurs passages éloignés d'un même auteur ; citons encore les références multiples au sein d'une même parenthèse ou dans l'évocation (souvent introduite par voir ou $c f$.) d'une liste d'auteurs. Assez proches de ces cas sont les résumés (conclusifs ou non) de travaux divers, comme dans cet exemple:

Dans les deux cas donc, un travail de recherche sur des évaluations plus informées, mieux instrumentées, aux cadres conceptuels affermis. [Br1]

Une autre série de marqueurs de métaphrase sont ceux qui signalent une appropriation du propos d'autrui par le scripteur : c'est nettement le cas de l'insertion d'une reprise dans une subordonnée, le propos d'autrui étant ainsi imbriqué dans une argumentation du scripteur:

Si nombre de disciplines telles que la docimologie ou la psychologie se sont intéressées à l'évaluation (Delcambre, 2013), les préoccupations liées à l'évaluation dans les didactiques disciplinaires sont particulièrement fortes et se trouveraient à la racine même de leur constitution (Martinand, 1996). [Br1]

Un marqueur assez proche est l'introduction d'une reprise par une expression telle que comme dirait $X$, dont le conditionnel met à distance l'énonciation de 
l'auteur (Le Querler, 1996) pour mieux intégrer le propos dans le discours du scripteur. Dans cette logique, assez curieusement, le commentaire métadiscursif en paraphrasant $X$ est un marqueur de métaphrase pour nous... Ainsi:

C'est aussi parce qu'au fond de l'activité de l'ingénieur il y a une activité de conception en paraphrasant Simon (1974). [Ar2]

D'autres marques de distance vont dans le sens de l'identification de la métaphrase, comme les modalisations dans la présentation d'une reprise:

L'évaluation désignerait tout d'abord dans son acception la plus large, « un acte social universel ». (Barbier, 1990 : 32) [BdL1]

Autre forme de distance, qu'on a déjà rencontrée : la référence à « ce que ne dit pas » le discours d'autrui ou les reprises assorties de commentaires polémiques ou, plus généralement, de jugements de valeurs (positifs ou négatifs) ou d'un soulignement par le scripteur du propos d'autrui.

Une troisième série de marqueurs consiste en la fusion du discours d'autrui dans celui du scripteur qui s'opère dans le changement de sens de connecteurs ou d'anaphores du discours cité quand il intègre un discours citant. Par exemple:

On pouvait ainsi lire dans les livrets de $6^{\text {ème }}$ que « si cette évaluation diagnostique permet[ait] d'analyser des savoir-faire et des difficultés à l'entrée au collège, elle ne [pouvait] être, parce qu'elle n'a[vait] ni la prétention ni la capacité d'être exhaustive, une évaluation bilan de l'école primaire ». (MEN, 2002, p. 2). [Br2]

L'anaphore cette, qui ouvre la citation, ne désigne pas exactement la même chose, puisque le cette renvoie à un cotexte qui n'est pas celui d'origine. Dans cet exemple, on observe un autre marqueur : la modification (signalée) de l'énoncé d'origine pour le faire s'insérer dans le cotexte du scripteur, assez proche de celui que nous avions vu plus haut (transformation de imbécile en imbécilité), qui consiste en la flexion d'un mot original pour mieux l'intégrer dans le cotexte du scripteur.

Inversement, indiquons quelques marqueurs de paraphrase : nous avons vu le discours direct libre ; on peut ajouter le discours indirect ou le discours indirect libre, ou encore les reprises introduites par d'après $X$, comme le dit $Y$, qui permettent au scripteur de fondre sa propre énonciation dans celle du discours repris (sans marque de distance ici). 
Un autre marqueur que nous avons vu est l'abandon de la forme première de l'énonciation du discours du scripteur au profit du système énonciatif du texte d'autrui : par exemple, quand un je ne renvoie pas au scripteur mais à un autre énonciateur ou quand les temps verbaux du discours du scripteur se calent sur ceux du discours repris.

Sont dits indécidables les énoncés où les deux modalités énonciatives, paraphrase et métaphrase, s'intriquent. Par exemple:

Or, selon Lavelle (1991 : 3), « le mot valeur s'applique partout où nous avons affaire à une rupture de l'indifférence ou de l'égalité entre les choses, partout où l'une d'elles doit être mise avant une autre ou au-dessus d'une autre, partout où elle est jugée supérieure et mérite de lui être préférée. » [BdL1]

Dans cet extrait, la citation ferait pencher à priori pour une paraphrase nette $(\mathrm{P}++)$, mais le « or » initial, qui insère le propos de l'auteur dans l'argumentation propre au scripteur, est une marque assez nette de métaphrase $(\mathrm{M}+$ ou $\mathrm{M}++) . \mathrm{La}$ citation entre guillemets est insérée dans un discours qui la digère énonciativement, ce qui rend indécidable la modalité à retenir pour cet énoncé dans son ensemble.

Pour finir, nous voulons souligner qu'il est, selon nous, nécessaire de distinguer formes et modalités des reprises d'autrui. En effet, même si certaines associations apparaissent vite (entre métaphrase et hybridation ou entre paraphrase et citation par exemple), il est intéressant d'être attentif aux dissociations possibles, comme dans le cas des citations métaphrastiques, où le discours cité est énonciativement modifié par le scripteur. Par exemple:

Anne-Marie Chartier (2003, p. 107), réfléchissant dans une perspective historique sur la culture scolaire à propos des exercices écrits et des cahiers d'élèves, conclut ainsi (c'est moi qui souligne) : «Selon le point de vue adopté, on peut focaliser le projecteur sur la rupture ou au contraire sur la continuité, mais le réformateur le plus radical ne parvient jamais à effacer en lui l'ancien élève. En matière de culture scolaire, s'il y a toujours un droit et un devoir d'inventaire, il n'y a pas de table rase possible. » [Dr1]

Cet exemple présente bien, sur le plan formel, une citation (guillemets, référence à un texte d'auteur), mais le scripteur l'insère dans son argumentation 
(par l'italique et la mention " je souligne ») : c'est là un effet assez clair de métaphrase.

Cette distinction entre modalités et formes de la reprise du discours d'autrui n'est pas souvent faite, mais nous la jugeons nécessaire et c'est ce qui fait sans doute l'originalité de notre approche.

\section{Résultats}

Nous présentons ici les résultats qui permettent d'identifier les différences entre les écrits de recherche et les écrits didactiques à partir des catégories que nous avons construites. Ces résultats concernent la moyenne des écrits produits : nous ne tenons pas compte ici des résultats pour chacun des scripteurs de notre corpus ; signalons simplement que les grandes tendances que nous identifions ici ne sont pas contredites par les résultats individuels (même si l'on peut observer quelques comportements un peu spécifiques sur certains aspects) ${ }^{18}$.

\section{Densité de reprise du discours d'autrui}

On observe en tout premier lieu ce qu'on pourrait appeler une plus grande densité de reprise dans les écrits de recherche que dans les écrits didactiques. Cela apparait dans le fait que, si les deux corpus sont à peu près équilibrés du point de vue du nombre de signes, le nombre de propositions où est visible une reprise du discours d'autrui (le nombre d'items retenus dans notre analyse du corpus complet) n'est pas le même, comme le montre le tableau ci-dessous:

18 Nous remercions Daniel Bart pour son aide dans le traitement statistique des données.

19 Nombre de signes des items retenus par rapport au nombre total de signes. 
Figure 2 - Nombres d'items selon les écrits

\begin{tabular}{|l|c|c|c|c|c|}
\cline { 2 - 6 } \multicolumn{1}{c|}{} & $\begin{array}{c}\text { Nombre } \\
\text { total } \\
\text { de signes }\end{array}$ & $\begin{array}{c}\text { Nombre } \\
\text { total } \\
\text { de mots }\end{array}$ & $\begin{array}{c}\text { Nombre } \\
\text { d'items } \\
\text { retenus }\end{array}$ & $\begin{array}{c}\text { Nombre } \\
\text { de signes } \\
\text { des items } \\
\text { retenus }\end{array}$ & Taux ${ }^{19}$ \\
\hline $\begin{array}{l}\text { Écrits } \\
\text { didactiques }\end{array}$ & 598573 & 91148 & 396 & 84716 & $14,15 \%$ \\
\hline $\begin{array}{l}\text { Écrits de } \\
\text { recherche }\end{array}$ & 658611 & 100343 & 941 & 246520 & $37,43 \%$ \\
\hline
\end{tabular}

Dans les écrits de recherche, plus de 37\% de la surface textuelle peut être considéré comme de la reprise de discours d'autrui (contre 14\% pour les écrits didactiques).

Si l'on prend en compte la nature des sources des reprises (rappelons que nous distinguons les discours issus d'auteurs académiques ou de données de recherche), on observe que le discours d'auteurs est plus nettement représenté dans les écrits didactiques que dans les écrits de recherche (le résultat est le même, qu'on calcule par énoncés ou par signes) : presque $84 \%$ des items sont issus de discours d'auteurs dans les écrits didactiques (contre 56\% dans les écrits de recherche). Concernant les discours issus des corpus de données, c'est l'inverse : ils sont plus présents dans les écrits de recherche (44\%) que dans les écrits didactiques (16\%). Ces résultats sont plus prononcés que ceux que nous avions présentés précédemment (DAUNAY, DELCAMBRE, 2016) à partir d'un corpus moins important.

On peut voir là une première différence entre les deux types d'écrits : les écrits didactiques accomplissent leur projet de formation en donnant une large place aux textes des auteurs jugés centraux pour l'acculturation des étudiants. $\mathrm{Au}$ contraire, les écrits de recherche donnent plus de place aux discours issus de la recherche empirique ; peut-être est-ce là une caractéristique disciplinaire : les sciences humaines, et en particulier les sciences de l'éducation et les didactiques, ont souvent des méthodologies de recherche basées sur le recueil et l'analyse de discours d'acteurs, enseignants et élèves/étudiants.

\section{Différences de modalités}

S'agissant de notre objet spécifique, les modalités des reprises, les différences sont également assez nettes (le calcul est fait à partir des énoncés) comme le montre le graphe ci-dessous. 
Figure 3 - Métaphrase et paraphrase selon les écrits

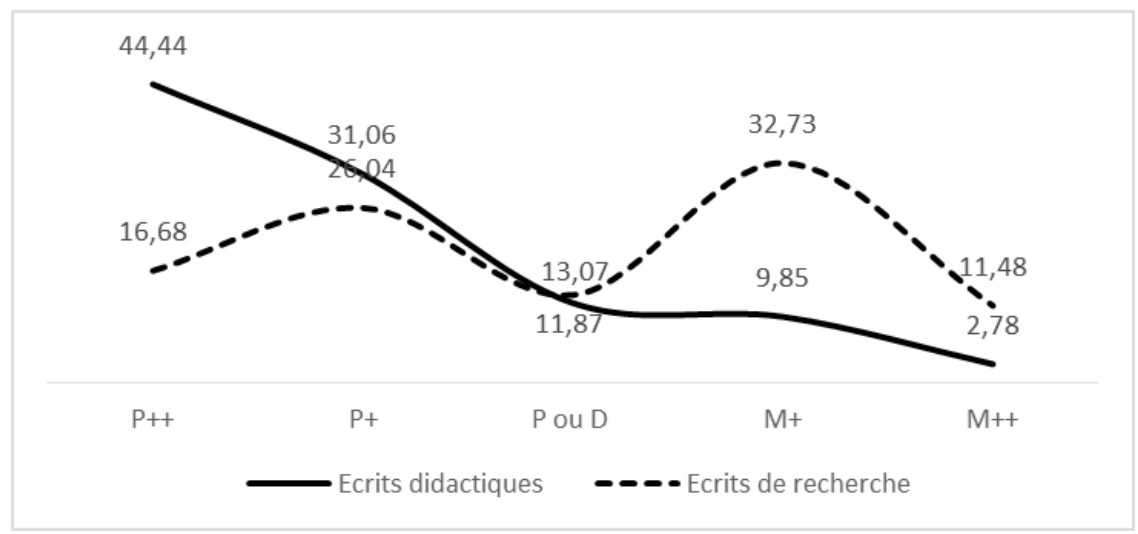

Ces résultats confirment en partie notre hypothèse. On voit que la part respective de la métaphrase ou de la paraphrase est différente selon les pratiques des enseignants-chercheurs : le taux d'énoncés tirant vers la paraphrase est plus grand dans les écrits didactiques que dans les écrits de recherche (avec un pic à $44,44 \%$ pour $\mathrm{P}++$ ) et c'est l'inverse pour le taux d'énoncés tirant vers la métaphrase (avec un pic à 32,73\% pour $\mathrm{M}+$ dans les écrits de recherche). Une autre analyse a été faite, qui va dans le même sens ; elle consiste à faire la moyenne des valeurs attribuées à chaque modalité (de 1 à 5) pour chaque type d'écrit : plus la moyenne est élevée, plus la métaphrase l'emporte. Or cette moyenne est de 1.95 pour les écrits didactiques et de 2.96 pour les écrits de recherche.

Cependant, la variable de la nature des reprises (issues d'auteurs ou de données de corpus) agit assez nettement, comme on le voit sur la figure 4, si l'on globalise les taux de paraphrase d'un côté, et de métaphrase de l'autre: 
Figure 4 - Taux des modalités polarisées

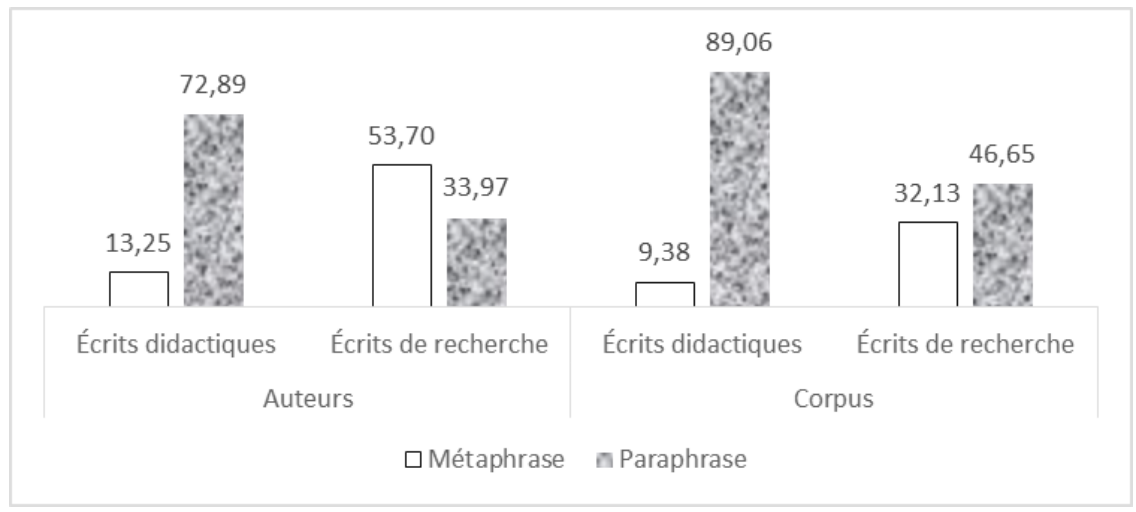

Ce graphique permet d'affiner le résultat précédent. Il montre que la domination de la métaphrase dans les écrits de recherche concerne en fait les reprises d'auteurs, et non les reprises d'éléments de corpus. Il montre aussi que la domination de la paraphrase dans les écrits didactiques vient autant (et même plus) des reprises de paroles issues de corpus que des reprises d'auteurs. Ce graphique fait ainsi apparaitre une nette différence entre les écrits didactiques et les écrits de recherche sur la question des modalités des reprises des discours d'autrui.

Cette différence est sans doute à mettre en lien avec les formes de reprises, qui sont également assez contrastées selon les écrits, comme le montre la figure 5:

Figure 5 - Taux des reprises selon leurs formes

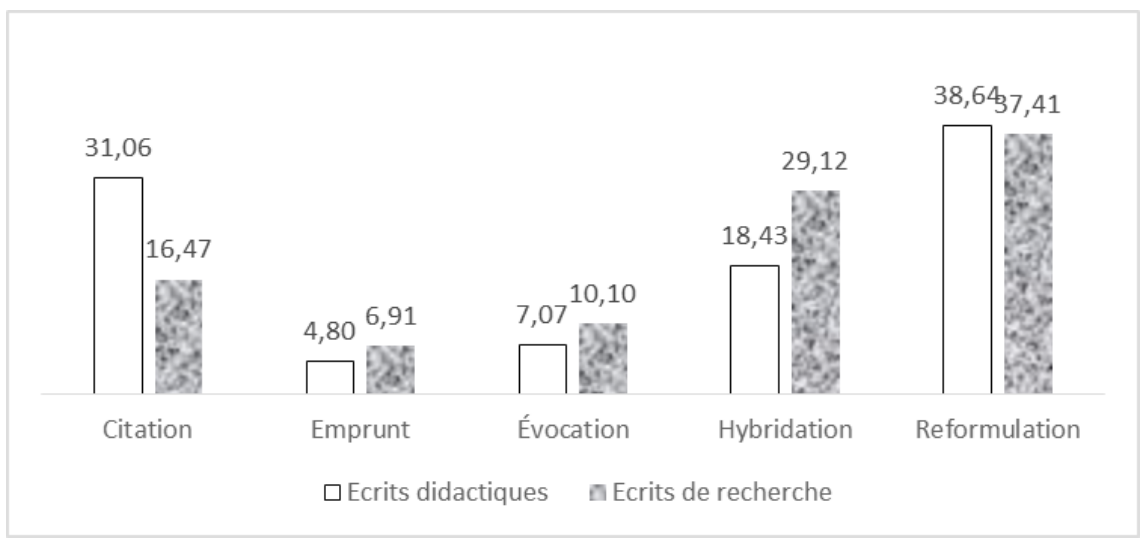

Ce graphique montre que les écrits de recherche utilisent plus que les écrits didactiques les formes de l'évocation et de l'hybridation, alors que les écrits 
didactiques utilisent plus que les écrits de recherche la forme de la citation, que l'on peut considérer comme plus didactique au sens où elle peut se prétendre plus fidèle à la lettre de l'énoncé cité et qu'elle suscite moins d'ambigüité ${ }^{20}$.

Cette distinction entre les écrits de recherche et les écrits didactiques est renforcée si on ne tient compte que des reprises d'auteurs : si les taux de reformulation de discours d'auteurs sont à peu près équivalents (environ $43 \%$ dans les deux cas), les écrits de recherche sont caractérisés par un taux plus important d'évocation ( $17 \%$ vs $8 \%$ ) et d'hybridation ( $26 \%$ vs 19\%), et les écrits didactiques par un taux bien plus important de citation (24\% vs 6\%).

Nous pouvons clore ces résultats en revenant sur le lien suggéré ci-dessus entre formes et modalités de la reprise du discours d'autrui. Nous avons établi, de fait, une forte solidarité entre la forme « citation » et la modalité " paraphrase » ou entre la forme « évocation » et la modalité « métaphrase ». Mais l'hybridation présente également une tendance métaphrastique et la reformulation une tendance paraphrastique (l'emprunt restant relativement peu marqué).

Ainsi la spécificité de notre approche, qui était de mieux distinguer formes et modalités, en postulant qu'elles ne recoupent pas exactement les mêmes réalités, se trouve-t-elle justifiée.

\section{Conclusion}

La dimension plus métaphrastique du discours de recherche, comme la place plus importante qu'il donne aux formes de l'évocation et de l'hybridation s'expliquent sans doute par le fait que « le discours scientifique est [...] construit comme un palimpseste, qui révèle autant qu'il masque les innombrables textes à partir desquels il se construit »(GROSSMANN, $\left.2010: \S 3^{21}\right)$.

20 Bien sûr, ce n'est ici qu'un effet de discours : comme le rappelle AUTHIER-REVUZ (1992, p. 38) le discours direct " n'est ni "objectif", ni "fidèle"; [...] même lorsqu'il cite textuellement - ce qui n'est pas nécessairement le cas, car sa propriété caractéristique est l'autonymie [...], non la textualité -, il ne peut pas pour autant être considéré comme "objectif" dans la mesure où reproduire la matérialité exacte d'un énoncé n'est pas restituer l'acte d'énonciation - dont l'énoncé est (n'est que) le "noyau" dans son intégralité ».

21 Cette remarque, faite dans un colloque, n'a pas été reprise dans sa version écrite (GROSSMANN, 2011). 
Les différences entre les deux types d'écrits mises en évidence dans cette étude seraient à interpréter vraisemblablement en termes d'enjeux sociaux différents, de prise en compte, dans l'écrit, de publics et d'attentes différents, comme l'ont bien montré BEACCO et DAROT (1984, p. 56-57). L'enseignant vise à diffuser le savoir construit par les auteurs qu'il considère essentiels à la formation de l'étudiant et à son entrée dans la culture disciplinaire qui est la sienne ; le chercheur vise plutôt à construire une argumentation scientifique, reposant sur les données qu'il a construites et intégrant dans son propre discours les discours d'autrui qui peuvent contribuer à lui donner une certaine autorité. Du reste, il serait intéressant d'enrichir la description que nous faisons là par un rapprochement avec les théories de l'argumentation, et particulièrement avec les approches des liens étroits qui peuvent apparaitre entre argumentation et discours rapportés (cf. VINCENT, DUBOIS, 1997 ; DOURY, 2004) ou encore avec les approches de l'effacement énonciatif ou de la co-, sous- et surénonciation (cf. GROSSMANN, RINCK, 2004, RABATEL, 2004).

C'était le sens de notre hypothèse, qui nous faisait supposer que le discours didactique donnerait plus de place à la parole des auteurs à faire découvrir aux étudiants alors que le discours scientifique mettrait plus en avant la discussion de ces auteurs, intriqués dans une argumentation du scripteur.

Mais ces différences ne doivent pas masquer leur relative proximité, qui s'explique par le fait qu'entre discours de recherche et d'enseignement, comme entre discours de recherche et de vulgarisation (cf. BEACCO, DAROT, 1984 ; JACOBI, 1986), les liens sont étroits : non seulement ce sont les mêmes personnes qui tiennent des rôles différents, mais ils sont l'un comme l'autre des discours de diffusion du savoir ; à cela s'ajoute que le discours didactique, en situation universitaire, a une fonction de modélisation du discours de recherche. Ce sont ces raisons qui expliquent les ressemblances entre les deux formes de discours académiques, et qui, en même temps, interrogent leurs sensibles différences. 


\section{Enunciative modalities of reported speech in written works of research and didactic texts Abstract}

The paper focuses on rewordings in academic writing. We make a comparison between two different faculty writing: didactic writing, developed for distance learning, and research writing for the scientific community. By enunciative modalities, we mean the ways a writer takes charge of the discourse of others which he takes up in his own discourse. After presenting our theoretical framework and terminology (including the identification of two poles of enunciative modalities we call paraphrase and metaphrase), we describe our corpus and methodology, before submitting the main results, among which the clearest one is that the respective share of paraphrase and metaphrase is different in teaching writing and research writing.

Keywords: Enunciation. Reported speech. Paraphrase. Metaphrase. Didactics. Academic Writing. 


\section{Références}

AUTHIER-REVUZ, Jacqueline. Hétérogénéité montrée et hétérogénéité constitutive : éléments pour une approche de l'autre dans le discours. DRLAV, Paris, n. 26, p. 91-151, 1982.

AUTHIER-REVUZ, Jacqueline. Repères dans le champ du discours rapporté. L'Information Grammaticale, Paris, n. 55, p. 38-42, 1992.

BAKHTINE, Mikhail. Esthétique et théorie du roman. Paris : Gallimard, 1934/1978.

BOCH, Françoise ; GROSSMANN, Francis. Se référer au discours d'autrui : comparaison entre experts et néophytes. Enjeux, Namur, n. 54, p. 41-51, 2002.

BOCH, Françoise ; RINK, Fanny. Pour une approche énonciative de l'écrit scientifique. Lidil, Grenoble, n. 41, p. 5-14, 2010.

BOUCHARD, Robert ; PARPETTE, Chantal. Autoportrait de l'enseignantchercheur en auteur/acteur. Jeu de postures et reformulations dans les cours magistraux de 1 1e année. Lidil, Grenoble, n. 35, p. 119-137, 2007.

BUSSMANN, Hadumod. Routledge Dictionary of Language and Linguistics. London-New York : Routledge, 1996.

CLAUDEL, Chantal. Pratiques citationnelles dans des cours magistraux à l'université. In : JAUBERT, Anna ; LÓPEZ MUÑOZ, Juan Manuel ; MARNETTE, Sophie ; ROSIER, Laurence; STOLZ, Claire (Org.). Citations II. Citer pour quoi faire ? Pragmatique de la citation. Louvain-la-Neuve : l'Harmattan-Academia, p. 189-205.

CRYSTAL, David. Dictionary of Linguistics and Phonetics. [S.l.] : Blackwell, 1980/1997.

DABÈNE, Michel. L'adulte et l'écriture. Contribution à une didactique de l'écrit en langue maternelle. Bruxelles : De Boeck-Wesmael, 1987.

DAUNAY, Bertrand. La paraphrase dans l'enseignement du français. Neuchâtel : Peter Lang, 2002. 
DAUNAY, Bertrand (Org.). Les écrits professionnels des enseignants. Approche didactique. Rennes : Presses Universitaires de Rennes, 2011.

DAUNAY, Bertrand. La métalepse du lecteur ou la porosité du métatexte. Cahiers de narratologie, Nice, 2017 à paraitre.

DAUNAY, Bertrand ; DELCAMBRE, Isabelle. Les modalités énonciatives de la reformulation Comparaison entre écriture d'enseignement et de recherche. Langues, cultures et sociétés, Kénitra, v. 2, n. 1, 2016. Disponible sur : $<\mathrm{http}: / /$ revues.imist.ma/? journal=LCS\&page=article\&op=view\&path $\% 5 \mathrm{~B} \% 5 \mathrm{D}=5783 \&$ path $\% 5 \mathrm{~B} \% 5 \mathrm{D}=3725>$. Accès le 09 fév. 2017.

DAUNAY, Bertrand ; DELCAMBRE, Isabelle ; DUFAYS, Jean-Louis ; THYRION, Francine (Org.). Didactique de l'écriture-lecture et formation des enseignants. Villeneuve d'Ascq : CEGES, 2007.

DELCAMBRE, Isabelle. Formes diverses d'articulation entre discours d'autrui et discours propre. Analyses de commentaires de textes théoriques. Lidil, Grenoble, n. 24, p. 135-166, 2001.

DELCAMBRE, Isabelle ; DONAHUE, Christiane. Academic Writing Activity: Student Writing in Transition. In : CASTELLÓ, Montserrat ; DONAHUE, Christiane (Org.). University Writing: Selves and Texts in Academic Societies. Bingley: Emerald Group Publishing Limited, 2012. p. 129-149.

DELCAMBRE, Isabelle ; LAHANIER-REUTER, Dominique. Proposition pour une étude sur les méthodes de recherche en didactique. Les Cahiers Théodile, Lille, n. 4, p. 123-142, 2003.

DOLIGNIER, Catherine. Plagiat, copie et reformulation paraphrastique dans l'écriture longue du mémoire de master. Mélanges CRAPEL, Nancy, v. 37, n. 1, p. 129-142, 2016.

DOMINICY, Marc. La naissance de la grammaire moderne. Langage, logique et philosophie à Port-Royal. Bruxelles : Mardaga, 1984. 
DOURY, Marianne. La fonction argumentative des échanges rapportés. In : LÓPEZ-MUÑOZ , Juan Manuel ; MARNETTE, Sophie ; ROSIER, Laurence (Org.). Le Discours rapporté dans tous ses états. Paris : L'Harmattan, 2004. p. 254-264.

DRILLON, Jacques. Traité de la ponctuation française. Paris : Gallimard, 1991. DUBOIS, Jean et al. Dictionnaire de linguistique et de sciences du langage. Paris : Larousse, 1994/2012.

FLECK, Frédérique. La possibilité d'un îlot. La répétition comme indice de littéralité. Linguistique et lexicographie latines et romanes, Paris, n. 11, 2015. Disponible sur : < http://www.paris-sorbonne.fr/IMG/pdf/DLL_11_F-Fleck.pdf>. Accès le 09 fév. 2017.

FLOREZ, Magda. La citation positionnée dans l'écrit scientifique, In : TUTIN, Agnès ; GROSSMANN, Francis (Org.). L'écrit scientifique. Du lexique au discours. Rennes : Presses universitaires de Rennes, 2013. p. 67-84.

FRIER, Catherine. Profils de lecteurs et modalités d'approche des textes de spécialité à l’université. Lidil, Grenoble, n. 17, p. 65-79, 1998.

GROSSMANN, Francis. Les modes de référence à autrui chez les experts : l'exemple de la Revue Langages. Faits de Langue, Berne, n. 19, p. 225-262, 2002.

GROSSMANN, Francis. Renvoyer aux sources du savoir: voir et $c f$. dans le texte scientifique. In: MELLET, Sylvie ; MARNETTE, Sophie ; LÓPEZ MUÑOZ, Juan Manuel ; ROSIER, Laurence. Communications du IVe Ci-dit, 2010. Disponible sur: http://revel.unice.fr/symposia/cidit/index.html?id=497> Accès le 21 août 2017.

GROSSMANN, Francis. Renvoyer aux sources du savoir : voir et $c f$. dans le texte scientifique. In : JAUBERT, Anna ; LÓPEZ MUÑOZ, 
